\title{
Isolation of Human Neutrophils from Whole Blood and Buffy Coats
}

\author{
Alan Y. Hsu ${ }^{1}$, Zhicheng Peng ${ }^{1}$, Hongbo Luo ${ }^{1}$, Fabien Loison ${ }^{1,2}$ \\ ${ }^{1}$ Department of Pathology, Harvard Medical School, Department of Lab Medicine, Children's Hospital Boston, and Dana-Farber/Harvard Cancer \\ Center ${ }^{2}$ Department of Microbiology, Faculty of Science, Mahidol University
}

\section{Corresponding Author}

Fabien Loison

fabien.loison@childrens.harvard.edu

\section{Citation}

Hsu, A.Y., Peng, Z., Luo, H., Loison, F. Isolation of Human Neutrophils from Whole Blood and Buffy Coats. J. Vis. Exp. (175), e62837, doi:10.3791/62837 (2021).

\section{Date Published}

September 17, 2021

DOI

$10.3791 / 62837$

URL

jove.com/video/62837

\section{Abstract}

Neutrophils (PMNs) are the most abundant leukocytes in human circulation, ranging from 40 to $70 \%$ of total blood leukocytes. They are the first cells recruited at the site of inflammation via rapid extravasation through vessels. There, neutrophils perform an array of functions to kill invading pathogens and mediate immune signaling. Freshly purified neutrophils from human blood are the model of choice for study, as no cell line fully replicates PMN functions and biology. However, neutrophils are short-lived, terminally differentiated cells and are highly susceptible to activation in response to physical (temperature, centrifugation speed) and biological (endotoxin, chemo- and cytokines) stimuli. Therefore, it is crucial to follow a standardized, reliable, and fast method to obtain pure and non-activated cells. This protocol presents an updated protocol combining density gradient centrifugation, red blood cell (RBC) sedimentation, and RBC lysis to obtain high PMN purity and minimize cell activation. Furthermore, methods to assess neutrophil isolation quality, viability, and purity are also discussed.

\section{Introduction}

The innate immune system is comprised of many cell types that maintain immune homeostasis and pathogen clearance along with many other physiological functions. Neutrophils comprise the largest pool of white blood cells in human circulation ${ }^{1}$. Most mature neutrophils are stored in the bone marrow, which is the site of generation for new neutrophils, also called granulopoiesis. In the bone marrow, granulocyte progenitors exit the cell cycle and terminally differentiate, acquiring their characteristic segmented nuclei and granules ${ }^{2}$. Under inflammatory conditions, in response to chemokines, cytokines, and damage-associated and pathogen-associated molecular patterns, neutrophils are mobilized from the bloodstream and out of the bone marrow to perform a wide array of functions. These include cytokine secretion, direct phagocytosis of the pathogen, the release 
of reactive oxygen species, degranulation of antimicrobial proteins, and formation of neutrophil extracellular traps.

The molecules used by neutrophils to fight infection are toxic to the microbes and the host. Thus, the life span and the proper removal of aging/dying neutrophils are highly regulated, and they have a limited life span in circulation $(<48$ $h)^{3}$. Due to this short survival, the human body produces on average 100 billion new neutrophils every day to maintain population homeostasis ${ }^{4}$. Emergency granulopoiesis can further increase the release of neutrophils, both mature and immature, in the blood during inflammation and infection ${ }^{5}$. The importance of neutrophils in the innate immune response is highlighted by patients with acquired or congenital neutropenia, who are susceptible to bacterial and fungal infections ${ }^{6}$.

Many challenges arise when studying neutrophil biology and their roles in the immune response due to their nature, including their short survival and cytotoxic content. Neutrophil-like cell lines have been commonly differentiated from human promyelocytic leukemia HL-60 cells and PLB-985 cells ${ }^{7,8}$. Although they can display neutrophil-like morphology and perform chemotaxis, these cell lines cannot fully recapitulate the biology of neutrophils. In vitro assays using these cell lines are also unable to recapitulate in vivo experiments. Furthermore, the differentiation of these cells needs to be induced and could be adversely affected by gene manipulation before differentiation.

Recently, methods have been developed to circumvent these issues by using inducible promoters to modulate gene expression post differentiation in HL-60 cells ${ }^{9}$. Even with such tools, primary human PMNs are required to validate targets using pharmacological approaches. Thus, it is imperative to obtain pure and inactivated neutrophils isolated from blood to validate the findings of cell line and animal models. This paper presents a revised PMN isolation protocol in which the pros and cons of current methods were evaluated ${ }^{10}$. A combination was devised consisting of gradient centrifugation to separate PMNs from other immune cells, short dextranbased sedimentation to remove the bulk of RBCs, rapid residual $\mathrm{RBC}$ lysis via osmotic pressure, and low-speed centrifugation to remove platelet contamination.

\section{Protocol}

NOTE: Human neutrophils were isolated from venous blood from discarded white blood cell filters obtained from the Blood Bank Lab at the Boston Children's Hospital. Blood donors were unidentifiable, and there was no interaction with living individuals or knowledge of identifiable personal information. Therefore, this work is not classified as human subjects research under the HHS human subjects regulations (45 CFR Part 46). The Boston Children's Hospital Institutional Review Board (IRB) approved the protocol.

\section{Layering the gradient}

1. After sterilizing the buffy coat or whole blood packaging and the laminar hood, divide the blood into $50 \mathrm{~mL}$ tubes with $10 \mathrm{~mL}$ of blood in each tube.

2. Bring the volume up to $35 \mathrm{~mL}$ with $5 \%$ fetal bovine serum (FBS)/Hank's Balanced Salt Solution (HBSS) to dilute the blood for a cleaner gradient.

NOTE: When using a leukapheresis membrane, cells can be flushed out using a $60 \mathrm{~mL}$ syringe and $30 \mathrm{~mL}$ of $5 \%$ FBS/HBSS per filter. Dilution is unnecessary when working with freshly drawn whole blood. 
3. Close the $50 \mathrm{~mL}$ tube lid, mix it several times by inversion, and keep it upside down to have the bottom devoid of RBCs.

4. Add $10 \mathrm{~mL}$ of density gradient medium (see the Table of Materials) directly beneath the blood. Ensure that the medium and the blood do not mix and that the interface is sharp (Figure 1A).

NOTE: This step is crucial. Ensure that the density gradient solution is at room temperature (RT) and wellmixed before each gradient. The first milliliter of the density gradient medium needs to be carefully and steadily layered as slowly as possible. Having the electronic pipette speed set to low is recommended.

5. Gently place the tube in a centrifuge without disturbing the gradient and spin at $400 \times g$ for 30 min at RT, making sure to disable the brake. Observe how the spun gradient separates into a top serum/plasma layer, a middle white ring of peripheral blood mononuclear cells (PBMCs), a cloudy density gradient medium layer, and a bottom pellet consisting of a white, thin neutrophil band on top of the RBCs (Figure 1B).

NOTE: A cloudy or opaque side of the tube after centrifugation can suggest that the cells (neutrophils) are activated and may not be suitable to use.

6. Remove the PBMCs first by emerging the suction pipette directly into the PBMC layer. Be sure to aspirate it completely while the serum/plasma layer decreases as the ring is removed. Scrape the side of the tube where cells pelleted with the suction pipette to maximize the removal of the PMBC. Carefully remove the cloudy density gradient medium layer between the PBMC ring and the neutrophil/RBC pellet.
NOTE: Scraping the pelleted cells on the side of the tube increases the purity of the isolation considerably. Be careful not to aspirate the pellet, as most neutrophils are sitting directly on top of the RBCs.

\section{Sedimentation of erythrocytes (RBCs)}

1. Using a $10 \mathrm{~mL}$ pipette, transfer the neutrophil/RBC pellet into a clean tube. Do not pipette up and down. Add 5\% FBS/HBSS to a final volume of $25 \mathrm{~mL}$. Gently mix by inversion.

NOTE: Performing the RBC sedimentation after the PBMC removal improves yield and decreases activation $^{10}$.

2. Directly add $25 \mathrm{~mL}$ of prewarmed $\left(37^{\circ} \mathrm{C}\right) \quad 3 \%$ Dextran $/ 0.9 \% \mathrm{NaCl} / \mathrm{H}_{2} \mathrm{O}$ into the tube containing the diluted neutrophil/RBC pellet and mix gently by inversion. Place the tube on a level, non-vibrating surface for 15 $\min$ (Figure 1C).

NOTE: Longer sedimentation reduces RBC contamination but also decreases the yield. Furthermore, prolonged dextran exposure may lead to neutrophil activation or cell death ${ }^{11}$.

3. Gently bring the tube back into the hood (for sterile isolation). By only slightly immersing the pipette in the liquid, collect the top layer $(\sim 30 \mathrm{~mL})$ following the liquid surface downwards.

NOTE: If the sedimentation produces a sharp interface between the medium and the RBCs, the RBC pellet is smaller, or if a higher yield is desired, collect up to $35 \mathrm{~mL}$.

4. Spin the tube $(400 \times g, 10 \mathrm{~min}, \mathrm{RT}$, using low brake (3)), resulting in a red pellet with no particles floating in the media. 


\section{Lysis of the residual RBCs}

1. Gently aspirate the supernatant without disrupting the pellet.

2. Add $25 \mathrm{~mL}$ of sterile ultrapure water directly into the tube and gently mix by inverting for $28 \mathrm{~s}$ to lyse the RBCs. Do not use a pipet to resuspend the pellet.

NOTE: Do not exceed $30 \mathrm{~s}$ as prolonged hypotonic conditions can activate and lead to neutrophil death ${ }^{12}$.

3. Immediately add $25 \mathrm{~mL}$ of sterile $1.8 \% \mathrm{NaCl} / \mathrm{H}_{2} \mathrm{O}$ into the tube and gently mix by inverting to bring the solution back to isotonic conditions.

NOTE: The solution should be red but without turbidity.

4. Spin down at $200 \times g$ for 3-5 min with low brake (level 3) to minimize RBCs and platelet sedimenting together with the neutrophils (Figure 1D and Figure 2) ${ }^{13,14}$.

NOTE: The pellet should be white with a minimal RBC layer on top, which can be gently removed while the supernatant is aspirated.

5. Resuspend neutrophils by pipetting the culture medium (10\% FBS/RPMI1640) directly on the pellet, but do not pipette up and down. Rock the tube horizontally from side to side to minimize cell activation.

NOTE: Cells should be kept at a concentration of $\sim 2 \times$ $10^{6}$ cells $/ \mathrm{mL}$, as higher density will lead to increased cell activation/death ${ }^{15}$. For the same reason, the cell pellet should be resuspended as soon as possible.

6. If cell aggregation or clumping is observed, filter the cell suspension using a $70 \mu \mathrm{m}$ mesh to discard clumped neutrophils.

\section{Determining neutrophil isolation quality}

1. Stain the cells with markers specific for neutrophils (CD66b, CD11b), eosinophils (CD193) (Figure 3), and an activation marker such as CD62L (Figure 4). Acquire 20,000 cells by flow cytometry. Analyze cell purity and activation using the gating strategies proposed in Figure 3 and Figure 4.

NOTE: The quality of the neutrophil preparation can be assessed using a 3\% acetic acid-methylene blue solution to visualize the unique lobed nucleus of neutrophils.

2. Determine cell viability using Annexin $\mathrm{V} /$ propidium iodide (PI) (Figure 5).

NOTE: Trypan blue staining can be used to evaluate cell viability.

\section{Representative Results}

When using density gradient to purify neutrophils, it is critical for the interface between the blood and the density gradient medium to be as sharp as possible, and that a distinct layer separation remains after centrifugation (step 1.4). Following RBC lysis, the buffer should be a clear red and not turbid (step 3.3). If the preparation is cloudy, a second round of lysis (step 3) may be required, although this may affect neutrophil survival (Figure 1). Following lysis, low-speed centrifugation $(200 \times g)$ is recommended when purity is prioritized, as it reduces platelet contamination considerably. However, high-speed centrifugation $(400 \times g)$ increases the yield at the expense of purity (step 3.4, Figure 2). After neutrophil isolation, fluorescence-activated cell sorting can be used to assess isolation purity (step 4.1) and should be chosen over microscopy. Although the FSC/SSC distribution of cells alone provides an estimate of the cell isolation quality (Figure 3A), the use of specific cell markers should be preferred. In 
this case, the most common contaminating cell populations are stained with specific antibodies together with CD66b, specifically expressed on granulocytes. CD45 staining is used to distinguish leukocytes (CD45+) and red blood cells and platelets (CD45-).

Other contaminants include lymphocytes (CD3+ or CD19+, Figure 3F), monocytes (CD14+, Figure 3D), and eosinophils (CD193+, Figure 3G). CD11b is an integrin expressed on the myeloid lineage; neutrophils and monocytes are CD11b+, whereas lymphocytes are CD11b- (Figure 3C).
As neutrophil activation can affect downstream experiments, the expression of CD62L should be assessed; neutrophils become CD62L- once activated (step 4.1). The peptide fMLP can be used as a positive control for CD62L shedding (Figure 4). It is also important to evaluate the health of neutrophils before performing assays; neutrophils have a relatively short half-life, and activation can further shorten it (step 4.2). A standard Annexin $\mathrm{V}$ and PI staining can give information on the live/dead status of the neutrophil culture at designated times (Figure 5).
(A)

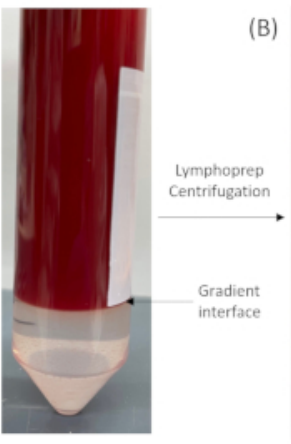

B)

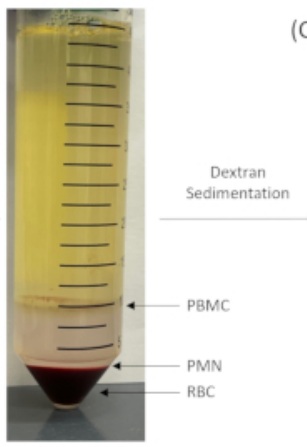

(C)

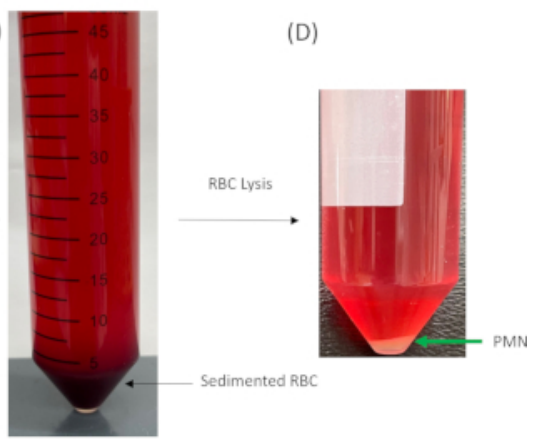

Figure 1: Density gradient medium-based separation of granulocytes. (A) Before and (B) after centrifugation. Note the sharp interface between the blood and the density gradient medium layers. (C) Sedimentation of the pellet in B resuspended (1:1) in 5\% FBS/HBSS and 3\% Dextran-0.9\% NaCl. (D) PMN pellet after lysis of the residual RBCs in the supernatant of (C) with $\mathrm{H}_{2} \mathrm{O}$. Abbreviations: $\mathrm{PBMC}=$ peripheral blood mononuclear cell; $\mathrm{PMN}=$ neutrophil; $\mathrm{RBC}=$ red blood cell; $\mathrm{FBS}=$ fetal bovine serum; HBSS = Hank's Balanced Salt Solution. Please click here to view a larger version of this figure. 
A

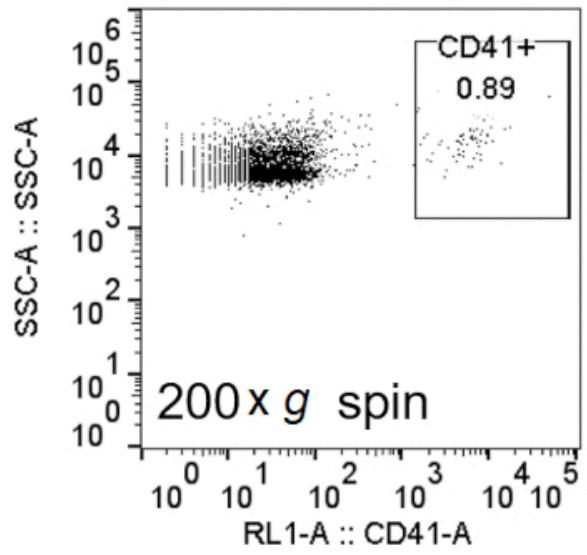

B

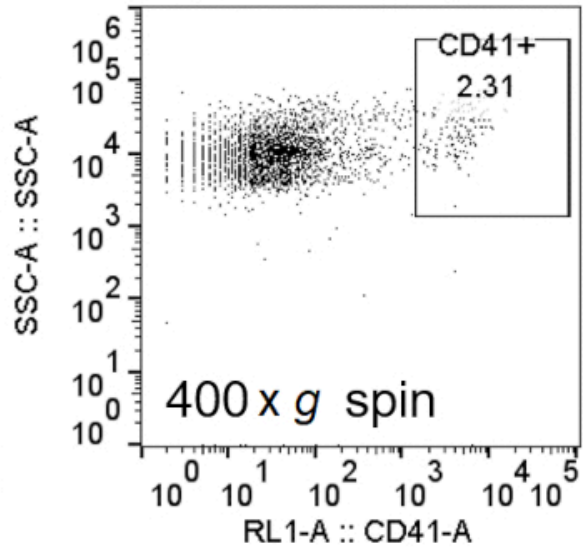

Figure 2: Spinning at lower speeds after RBC lysis decreased platelet contamination. After lysis of $\mathrm{RBCs}$ with $\mathrm{H}_{2} \mathrm{O}$, cells were spun down at $200 \times g($ A $)$ or $400 \times g(B)$. Neutrophils were stained, and flow cytometry analysis was performed, as described in Figure 3, with the addition of anti-CD41 to label the platelets. Abbreviations: RBC $=$ red blood cell; $C D 41=$ cluster of differentiation 41; SSC-A = side scatter-Area. Please click here to view a larger version of this figure. 


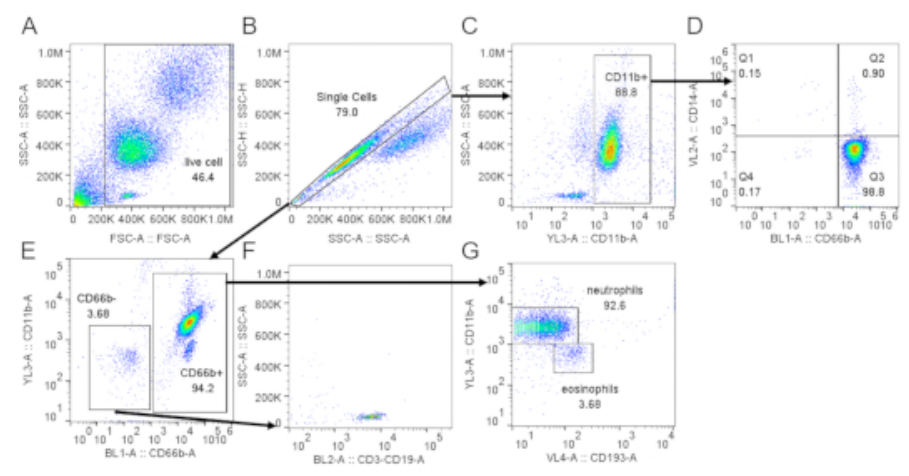

$\mathrm{H}$

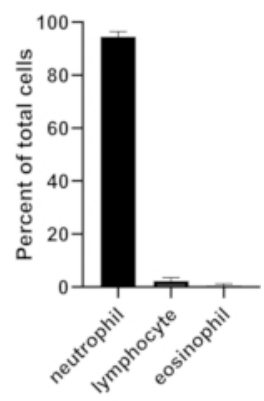

Figure 3: Assessment of neutrophils isolated from buffy coat. Isolated neutrophils were stained with anti-CD66b, anti-CD11b, anti-CD14, and anti-CD193 following a standard protocol. Single cells (B) were gated from total cells (A). (C) $\mathrm{CD}_{11 \mathrm{~b}^{+}}$cells were gated from single cells. (D) Dot plot showing neutrophils (CD66b ${ }^{+}, \mathrm{CD} 14$ low/-) and a very low

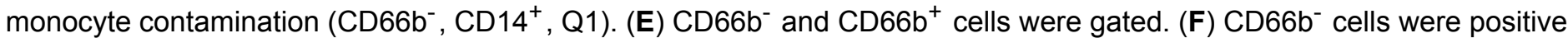
for lymphocyte markers (CD3, CD19). (G) Expression of CD11b and CD193 in CD66b ${ }^{+}$cells, showing distinct neutrophil $\left(\mathrm{CD} 6 \mathrm{~b}^{+}, \mathrm{CD} 11 \mathrm{~b}^{+}, \mathrm{CD} 193^{-}\right)$and eosinophil $\left(\mathrm{CD} 6 \mathrm{~b}^{+}, \mathrm{CD} 11 \mathrm{~b}^{-}, \mathrm{CD} 193^{+}\right)$populations. In the representative result shown here, neutrophil purity is $\sim 93 \%$ with $\sim 3.7 \%$ lymphocyte and $\sim 3.7 \%$ eosinophil contamination. (H) Quantification of neutrophil purity after purification. Data are compiled from 5 individual trials and presented as mean $\pm \mathrm{SD}$. Abbreviations: CD $=$ cluster of differentiation; SSC-A = SSC-A = side scatter-Area; FSC-A = forward scatter-Area. Please click here to view a larger version of this figure. 

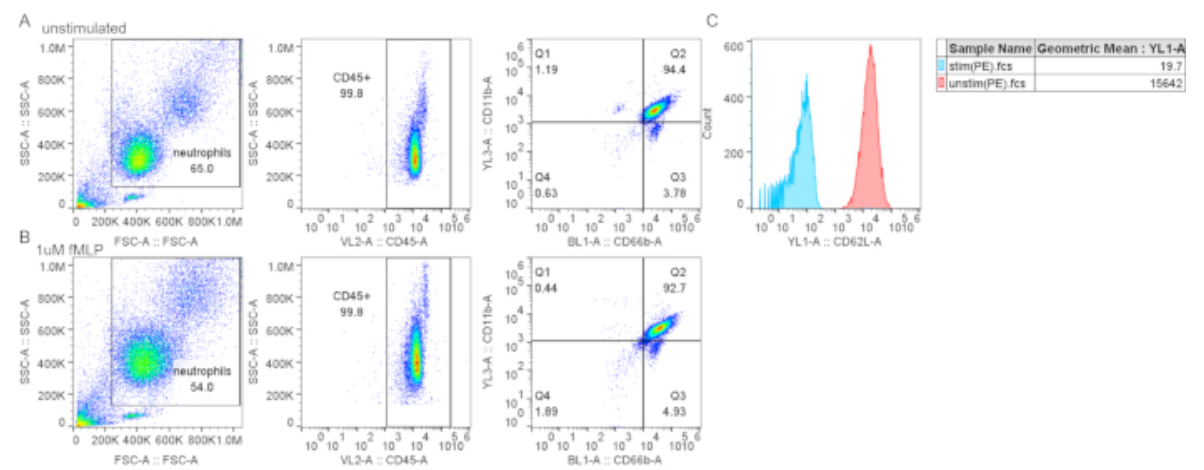

Figure 4: Gradient purification did not cause CD62L shedding. (A-B) Control (A) or fMLP-stimulated neutrophils (B) (1 $\mathrm{mM}$ fMLP for $15 \mathrm{~min}$ at $37^{\circ} \mathrm{C}$ ) were stained with neutrophil markers and CD62L. (C) Fluorescent mean intensity for CD62L is decreased in fMLP-treated cells, indicating CD62L shedding and neutrophil activation. Abbreviations: CD26L $=\mathrm{L}-\mathrm{selectin}$; fMLP = N-Formylmethionyl-leucyl-phenylalanine; SSC-A = SSC-A = side scatter-Area; FSC-A = forward scatter-Area; PE = phycoerythrin. Please click here to view a larger version of this figure.
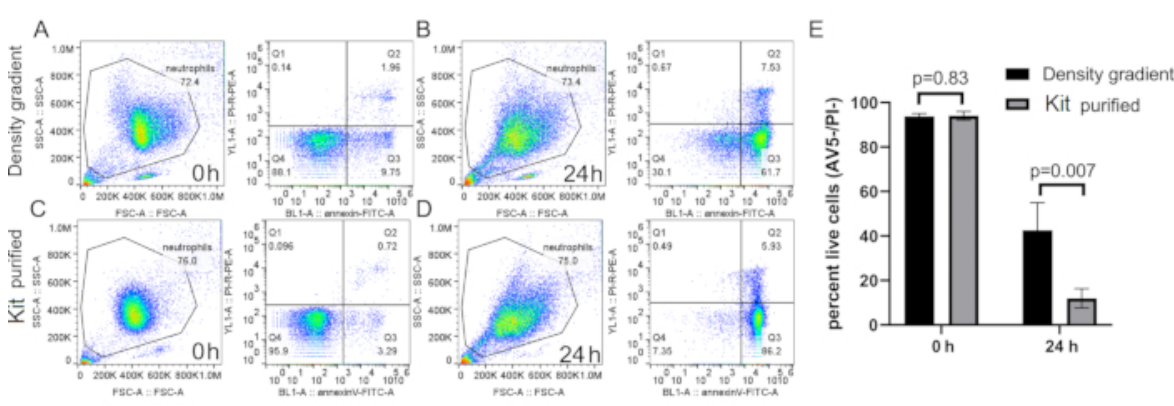

Figure 5: Spontaneous death of neutrophils purified by density gradient or neutrophil isolation kit. Neutrophils were purified with density gradient (A and B) or commercial microbeads (C and $\mathbf{D})$ and cultured for $0 \mathrm{~h}(\mathbf{A}$ and $\mathbf{C})$ or $24 \mathrm{~h}(\mathbf{B}$ and D) in RPMI-10\% FCS. Cells were stained using Annexin V and PI at respective time points following a standard protocol. (E) Quantification of purified neutrophil spontaneous death. $n=5$, mean \pm SD. Abbreviations: SSC-A $=S S C-A=$ side scatterArea; FSC-A = forward scatter-Area; PI = propidium iodide; FCS = fetal calf serum; FITC = fluorescein isothiocyanate; $A V 5$ = Annexin V. Please click here to view a larger version of this figure.

\section{Discussion}

Due to the short life span, terminal differentiation status, and lytic content of neutrophils, studying these cells has always been a challenge. Apart from utilizing mouse models or cells from patient cohorts, cell lines are useful tools to help study neutrophil biology ${ }^{16}$. However, neutrophil-like cell 
lines cannot completely reiterate all aspects of neutrophil biology, adding an extra layer of difficulty in studying these cells. The most commonly used in vitro model is the HL-60 cell line, which can be differentiated into neutrophil-like cells by treatment with dimethylsulfoxide or retinoic acid 17,18 . Although these cells are useful in the study of migration and respiratory burst, they are not suitable for studying the microbiocidal activity of neutrophils. Other cell lines exist (PLB-98, NB4), and they are also associated with their set of limitations ${ }^{19}$.

It is pivotal to validate with primary human neutrophils observations made with mouse disease models and cell lines. Neutrophils cannot be cryopreserved efficiently and thus are often freshly isolated from whole blood or buffy coats obtained from donors and immediately processed. Once isolated, the cells start to undergo a complex form of spontaneous death, regulated by oxidation, cytoplasmic caspases, and proteases found in neutrophil granules 20,21 . Improper isolation methods or techniques can lead to the activation of neutrophils, only accelerating cell demise. It is imperative to have a reliable and consistent method to obtain pure and high-quality neutrophils from donors.

There have been many methods published on human neutrophil isolation ${ }^{10,22}$. They mainly fall into two categories, with some shared strategies. The first category is antibodybased, being either through positive or negative selection. Positive selection would label neutrophils directly, therefore providing a highly pure cell population, although it also leads to rapid cell activation, cell death, as well as unwanted tagging of neutrophils ${ }^{23}$. Negative selection, though leaving the cells unlabeled and giving a very pure population, accelerated neutrophil death, although the precise mechanism is unknown (Figure 5). Whether gene or protein expression is also altered after positive or negative selection needs to be further investigated. Moreover, due to the amount of antibody needed to deplete the other types of cells, these methods cannot output large amounts of neutrophils. However, antibody-based assays can still be used for shortterm culture and experiments on a smaller scale and are methods of choice for experiments requiring very high cell purity, such as gene and protein expression studies.

The second type of isolation method is gradient- and density-based. It usually involves Percoll, Ficoll-Paque, or other polysaccharide/polyvinylpyrrolidone components and utilizes centrifugation force to separate different types of blood cells based on cell density. These methods often are complemented with the sedimentation of red blood cells by dextran. These methods can handle larger scales of starting material and can achieve high purity as well. One caveat of density-based isolation is the inefficient separation of other far less abundant granulocytes (mostly eosinophils) from neutrophils, and it is, therefore, the major limitation of the protocol presented, as even the presence of small cell contamination can affect neutrophil response ${ }^{24}$.

Presented here is a summarized method based on gradient isolation, refining previous methods ${ }^{10,22}$. We utilize the current understating of neutrophil specificities to reliably isolate pure human neutrophils with limited residual platelet and RBCs, preventing neutrophil activation and accelerated death. The most crucial step is the layering of the gradient, which is much more effectively obtained by adding the density gradient medium underneath the blood to obtain a sharp interface. A quick examination of the PBMC ring and the gradient after centrifugation can reveal possible contamination, activation, and low yields. When working with a leukapheresis membrane or buffy coat, diluting the blood 
is important as excessive cell density would lead to cell aggregation, leading to impurities and cell activation.

This protocol should be completed within $2 \mathrm{~h}$ to ensure cell freshness, and steps involving the density gradient medium, dextran, and lysis be done immediately, as exposure to these solutions can alter neutrophils. With this protocol, the expected yield of neutrophils is at least $\sim 10$ million $/ 10 \mathrm{~mL}$ of whole blood and at least $\sim 60$ million/10 $\mathrm{mL}$ of buffy coat. Evaluation of isolation quality should be done as follows: activated neutrophils should be less than $10 \%$, lymphocyte contamination lower than $5 \%$, minimal eosinophil (same side scatter but lower forward scatter population), and cell viability should be over $90 \%$. Lower purity can result from improper layering or storage of the density gradient medium or due to the quality and freshness of the starting blood product.

\section{Disclosures}

The authors declare that the research was conducted in the absence of any conflict of interest.

\section{Acknowledgments}

This Project was supported by P01HL095489. A.Y.H was supported by T32HL066987.

\section{References}

1. Rosales, C. Neutrophil: A cell with many roles in inflammation or several cell types? Frontiers in Physiology. 9, 113 (2018).

2. Amulic, B., Cazalet, C., Hayes, G. L., Metzler, K. D., Zychlinsky, A. Neutrophil function: from mechanisms to disease. Annual Review of Immunology. 30, 459-489 (2012).
3. Stoller, J. K. Murray \& Nadel's textbook of respiratory medicine, 6th edition. Annals of the American Thoracic Society. 12 (8), 1257-1258 (2015).

4. Dancey, J. T., Deubelbeiss, K. A., Harker, L. A., Finch, C. A. Neutrophil kinetics in man. Journal of Clinical Investigation. 58 (3), 705-715 (1976).

5. Manz, M. G., Boettcher, S. Emergency granulopoiesis. Nature Reviews. Immunology. 14 (5), 302-314 (2014).

6. Summers, C. et al. Neutrophil kinetics in health and disease. Trends in Immunology. 31 (8), 318-324 (2010).

7. Pedruzzi, E., Fay, M., Elbim, C., Gaudry, M., GougerotPocidalo, M. A. Differentiation of PLB-985 myeloid cells into mature neutrophils, shown by degranulation of terminally differentiated compartments in response to $\mathrm{N}$-formyl peptide and priming of superoxide anion production by granulocyte-macrophage colonystimulating factor. British Journal of Haematology. 117 (3), 719-726 (2002).

8. Tucker, K. A., Lilly, M. B., Heck, L., Rado, T. A. Characterization of a new human-diploid myeloidleukemia cell-line (Plb-985) with granulocytic and monocytic differentiating capacity. Blood. 70 (2), 372-378 (1987).

9. Hsu, A. Y. et al. Inducible overexpression of zebrafish microRNA-722 suppresses chemotaxis of human neutrophil like cells. Molecular Immunology. 112, 206-214 (2019)

10. Kremserova, S., Nauseef, W. M. Isolation of human neutrophils from venous blood. Methods in Molecular Biology. 2087, 33-42 (2020).

11. Quach, A., Ferrante, A. The application of dextran sedimentation as an initial step in neutrophil purification 
promotes their stimulation, due to the presence of monocytes. Journal of Immunological Research. 2017, 1254792 (2017).

12. Thorson, L. M., Turkalj, A., Hung, J. C. In vitro evaluation of neutrophil viability after exposure to a hypotonic medium. Nuclear Medicine Communications. 16 (7), 615-620 (1995).

13. Dhurat, R., Sukesh, M. Principles and methods of preparation of platelet-rich plasma: a review and author's perspective. Journal of Cutaneous and Aesthetetic Surgery. 7 (4), 189-197 (2014).

14. Etulain, J. et al. An optimised protocol for plateletrich plasma preparation to improve its angiogenic and regenerative properties. Scientific Reports. 8 (1), 1513 (2018).

15. Hannah, S. et al. Constitutive neutrophil apoptosis in culture is modulated by cell density independently of beta2 integrin-mediated adhesion. FEBS Letters. 421 (2), 141-146 (1998).

16. Hsu, A. Y. et al. Phenotypical microRNA screen reveals a noncanonical role of CDK2 in regulating neutrophil migration. Proceedings of the National Acadermy of Sciences of the United States of America. 116 (37), 18561-18570 (2019).

17. Martin, S. J., Bradley, J. G., Cotter, T. G. HL-60 cells induced to differentiate towards neutrophils subsequently die via apoptosis. Clinical and Experimental Immunology. 79 (3), 448-453 (1990).

18. Hauert, A. B., Martinelli, S., Marone, C., Niggli, V. Differentiated HL-60 cells are a valid model system for the analysis of human neutrophil migration and chemotaxis. International Journal of Biochemistry and Cell Biology. 34 (7), 838-854 (2002).
19. Blanter, M., Gouwy, M., Struyf, S. Studying neutrophil function in vitro: Cell models and environmental factors. Journal of Inflammation Research. 14, 141-162 (2021).

20. Kambara, H. et al. Gasdermin D exerts anti-inflammatory effects by promoting neutrophil death. Cell Reports. 22 (11), 2924-2936 (2018).

21. Loison, F. et al. Proteinase 3-dependent caspase-3 cleavage modulates neutrophil death and inflammation. Journal of Clinical Investigation. 124 (10), 4445-4458 (2014).

22. Siemsen, D. W. et al. Neutrophil isolation from nonhuman species. Methods in Molecular Biology. 1124, 19-37 (2014).

23. Hasenberg, M. et al. Rapid immunomagnetic negative enrichment of neutrophil granulocytes from murine bone marrow for functional studies in vitro and in vivo. PLoS One. 6 (2), e17314 (2011).

24. Calzetti, F., Tamassia, N., Arruda-Silva, F., Gasperini, S., Cassatella, M. A. The importance of being "pure" neutrophils. Journal of Allergy and Clinical Immunology. 139 (1), 352-355 e356 (2017). 